\title{
PROBLEMS FOR SOLUTION
}

P 66. "Gauss' Lemma"( $\$ 23$, vol. 1 of Modern Algebra by Van der Waerden) is essentially equivalent to the statement that a unique factorization domain $R$ has the following property:

(*) $\left\{\begin{array}{l}\text { If } K \text { is the field of quotients } \\ \text { of } R \text {, then a polynomial over } R \\ \text { which factors over } K \text { factors over } R .\end{array}\right.$

Show that the following converse holds: if $R$ is a domain in which every element can be expressed as a product of irreducible elements - for example if $R$ is Noetherian - and if $R$ has property (*), then $R$ is a unique factorization domain.

Carl Riehm, McGill University

P 67. Let

$$
C=\lim _{n \rightarrow \infty}\left[\sum_{j=1}^{n} \frac{1}{j}-\ln n\right]
$$

denote the Euler-Mascheroni constant and let $\mathbf{x}$ be a real variable.

Determine the following limit:

$$
\lim _{x \rightarrow 0} x^{-2}\left\{C+\mathcal{K}\left(\Gamma^{\prime}(i x) / \Gamma(i x)\right)\right\}
$$

$R=$ real part of.

H. G. Helfenstein, University of Ottawa

P 68. Find all solutions of

$$
\phi\left(2^{2^{n}}-1\right)=\phi\left(2^{2^{n}}\right)
$$

where $\phi$ is Euler's function.

David Klarner, University of Alberta 
P 69. It is a familiar fact that a cyclic permutation of length $\bar{n}$ can be written as a product of $n-1$ transpositions. Show that it cannot be done so more economically.

I. Connell, McGill University

P 70. Prove that every finite abelian group is isomorphic to a subgroup of the multiplicative group of integers relatively prime to $m, \bmod m$, for suitable $m$.

Carl Riehm, McGill University 